En résumé la stérilisation du lait par la chaleur doit encore actuellement tenir sa place dans la fabrication des produits lactés ; mais, si elle devait un jour disparaître, ce que l'avenir seul nous dira, pour être remplacée par un procédé qui lui serait supérieur, il n'y aurait évidemment pas à le regretter, mais il faudrait néanmoins convenir que cette stérilisation aura joué un rôle réellement utile pendant un grand nombre d'années.

Qu'il nous soit enfin permis ici de remereier tout particulièrement MM. les Drs Hallion, Robin et Gaillet qui, à différentes reprises, ont bien voulu nous favoriser de leurs précieux conseils ou de leurs judicieuses observations, ainsi que M. Porlier, Docteur en droit, administrateur de la Société laitière Carrion, qui, également spécialiste des questions laitières, a bien voulu mettre à notre disposition les moyens matériels pour réaliser nos essais.

\title{
BIBLICGRAPHIE
}

LINDEx ( $\mathrm{Le}$ lait, la erème, le beurre et les fromages);

DoRnic et Choluet (Lait, beurre et dérivés);

Datre (Les microbes du lait);

Seligman (Comptes rendus de la conférence nationale sur la pasteurisation du lait - Londres 1923); traduction in Le Lait, 1926;

Porcher (Conférences à l'Institut Pasteur, Novembre-Décembre 1923);

GutTtonnead (Les principes d'une technique rationnelle en industrio laitière)

Le Lait, 1924-1925;

Gorgerat (Procédé de stérilisation du lait en vase clos sans autoclave) Le Lait; ORLA-JENSEN (The milk industry);

Stassano (De la pasteurisation) Le Lait, 1926 ;

Dr RoBrn (Technique de la stérilisation du lait), Le Lail, janvier 1927;

Monvoisix (Le lait, - Physiologie, analyse, utilisation).

\section{UNE MODIFICATION DE LA MICRO-MÉTHODE DE LINDNER POUR LES ESSAIS DE FERMENTATION}

\author{
Par Assen KANTARDJIEFF,
}

Assistant à la Faculté agronomique de l'Université de Sofia.

On sait que lors de l'étude de la physiologie des microorganismes on attache avec raison une grande importance à la détermination du pouvoir fermentatif vis-à-vis de divers hydrates de carbone. C'est ainsi que l'on reconnait par exemple certaines levures, certaines bactéries lactiques ou autres à leur pouvoir de fermenter certains sucres. L'exécution de l'épreuve fermentative exacte a cependant ses difficultés. Il faut tout d'abord disposer d'un milieu de culture de base bien adapté à l'espèce microbienne que l'on veut soumettre à l'épreuve, débarrassé 
complètement des sucres qu'il pourrait contenir et exactement neutralisé. La préparation de ce milieu exige beaucoup de soins, de temps et de matériaux. En outre la stérilisation du milieu de base, des solutions de sucre et éventuellement de la solution de tournesol, qui doivent se faire séparément, prennent également du temps. L'addition des solutions de sucres et de tournesol au milieu de base sous des conditions d'asepsie rigoureuse est aussi une opération délicate. Tout cela contribue à rendre la détermination des propriétés physiologiques des microorganismes longue et délicate et, vu les très hauts prix de certains sucres, très coûteuse. Dans bien des cas il est nécessaire, dans les laboratoires de bactériologie technique, de disposer d'une méthode plus rapide et moins chère et c'est pour répondre à ce besoin que LINDNER a imaginé sa micro-méthode pour les essais de fermentation. Voici en quoi consiste ce procédé trop peu connu.

$1^{0}$ Stérilisation dans la flamme d'une lamelle et d'une lame porteobjets excavée, comme on l'utilise pour les préparations dans la goutte suspendue. La lamelle est ensuite placée sur la lame, à eôté de l'excavation.

$2^{\circ}$ Mettre 2 ou 3 gouttes d'un milieu de base approprié, et s'il s'agit de levures, simplement 2 ou 3 gouttes d'eau stérilisée dans l'exeavation de la lame.

$3^{\circ}$ Ajouter une petite anse du sucre sur lequel on veut éprouver les microorganismes.

$4^{\circ}$ Ensemencer ces derniers à dose massive; faire glisser la lamelle sur l'excavation de la lame, en veillant à ce qu'il ne reste pas de bulles d'air dans l'interstice.

$5^{\circ}$ Eloigner le surplus de liquide qui se trouve sur la lame au moyen d'un petit carré de papier buvard stérilisé.

$6^{\circ}$ Sceller les bords de la lamelle à la lame avec de la vaseline liquéfiée a fin d'empêcher le liquide de la cavité de se dessécher.

$7^{\circ}$ Porter la préparation à l'étuve et laisser travailler les microorganismes.

La formation de bulles de gaz indique la fermentation du sucre addi. tionné, si l'on a travaillé proprement et si l'on contrôle encore la pureté de la culture à la fin de l'expérience on peut être sûr des résultats obtenus. Ce procédé rend d'excellents services dans bien des cas. Cependant il existe une foule de réactions microbiennes qui ne se traduisent pas par une fermentation avec dégagement de gaz. Nous rappelons simplement que ce que l'on convient de nommer fermentation lactique est une transformation du sucre en acide lactique sans production de gaz. L'absence de dégagement gazeux ne prouve donc pas qu'il n'y ait pas eu fermentation. C'est pour obvier à cet inconvénient que HENNEBERG recommande d'ajouter un peu de craie stérilisée au liquide, à part le sucre. S'il y a production d'acide, ce dernier se substituera à l'acide 
earbonique de la craie en le libérant. La formation d'acide se traduira done par une formation de gaz. Ce procédé a cependant l'inconvénient de ne pas donner de différence entre les microbes qui produisent seulement de l'acide et ceux qui font fermenter avec production de gaz.

Afin de parer à eet état de choses nous avons eu l'idée de remplacer la craie recommandée par HENNEBERG par un peu de solution de tournesol. On peut alors observer si le microbe étudié produit du gaz et reconnaître au rougissement du liquide s'il produit de l'acide aux dépens d'un sucre.

L'addition de solution de tournesol stérilisée doit être faite avec prudence. Si l'on en met trop, les microbes en souffrent et ne se développent pas. L'addition du tournesol peut se faire soit directement dans le liquide de l'excavation en y ajoutant une anse d'une solution concentrée, soit, si l'on travaille en séries, dans le milieu de base après sa stérilisation.

La simplicité du procédé de LINDNER amélioré en fait une méthode facile. En quelques minutes on apprendra à s'en servir correctement. On obtiendra ainsi de bons résultats aveo des frais très restreints.

\section{DOSAGE DE LA MATIÈRE GRASSE dANS LA CASÉINE INDUSTRIELLE}

\author{
par A. TAPERNOUX, \\ Chef de travaux de Chimle à l'Ecole \\ Vétérinaire de Lyon
}

\section{R. DESRANTE,}

Ancien élève de l'Ecole de Laiterie de Surgères

et J. BINEAU, Ingénieur agricole (E.N.G.).

La matière grasse constitue une impureté de la caséine que les méthodes actuelles d'écrémage ne permettent pas d'éliminer d'une façon parfaite. Il est en effet pratiquement difficile d'obtenir un lait écrémé ne contenant plus que des traces de matière grasse et dans la majorité des cas, il reste dans le lait de 0 gr. 50 à 1 gr. de matière grasse par litre, après l'éerémage.

Il est intéressant de pouvoir doser facilement la matière grasse d'une caséine, a fin de s'assurer du bon écrémage du lait qui a servi à sa préparation et d'autre part de renseigner les industries qui utilisent le produit et dans lesquelles la teneur en matière grasse joue un rôle.

Nous nous proposqns de décrire deux méthodes que nous avons employées comparativement et qui possèdent sur celles décrites jusqu'à présent l'avantage de la rapidité. La méthode de ScHMidt-BondzynSKI a le défaut d'être assez longue surtout par le temps de repos nécessité pour la séparation de la couche limpide. 